\title{
Imagen en pericarditis constrictiva: reporte de tres casos
}

\author{
Denisse Guzmán Ramírez \\ Arcenio Lendo López \\ Anival Trujillo García
}

\section{Correspondencia}

Denisse Guzmán Ramírez

draguzman@cardiologiamonterrey.com.mx

Servicio de Cardiología. Hospital de Cardiología UMAE 34. Instituto Mexicano del Seguro Social. Monterrey (Nuevo León). México

Recibido: 12/02/2020

Aceptado: 21/10/2020

En línea: 31/12/2020

Citar como: Guzmán Ramírez D, Lendo López A, Trujillo García A. Imagen en pericarditis constrictiva: reporte de tres casos. Rev Ecocar Pract (RETIC). 2020 (Dic); 3 (3): 1-5. doi: 10.37615/retic.v3n3a5.

Cite this as: Guzmán Ramírez D, Lendo López A, Trujillo García A. Image in constrictive pericarditis: report of three cases. Rev Ecocar Pract (RETIC). 2020 (Dic); 3 (3): 1-5. doi: 10.37615/retic.v3n3a5.

\section{Palabras clave \\ $\triangleright$ Pericarditis constrictiva \\ $\triangleright$ Calcificación pericárdica \\ $\triangleright$ Insuficiencia cardíaca \\ $\triangleright$ Disfunción diastólica}

\section{Keywords \\ $\triangleright$ Constrictive pericarditis \\ $\triangleright$ Pericardial calcification \\ $\triangleright$ Heart failure \\ $\triangleright$ Diastolic dysfunction}

\section{Caso clínico 1}

Varón de 46 años con edema de miembros inferiores, disnea de grandes esfuerzos, disnea paroxística nocturna y ortopnea de 6 meses de evolución, con historia de exposición a silicato de magnesio (talcos) durante 5 años, tabaquismo y alcoholismo, e hipertensión arterial sistémica.

En el ecocardiograma transtorácico (ETT), se observaba un pericardio calcificado de $4 \mathrm{~mm}$ de grosor, separación de hojas pericárdicas de $7 \mathrm{~mm}$ con contenido sólido en su interior y rebote (bounce) septal, encontrándose la fracción de eyección ventricular izquierda conservada (62\%) (Vídeo 1); función diastólica izquierda con patrón de alteración de la relajación, annulus reversus, variación respiratoria del flujo transmitral del 54\% y variación respiratoria del flujo transtricuspídeo del 62\% (Figura 1) y, finalmente, flujo diastólico reverso predominante en vena hepática (Figura 2).

Se realizó tomografía computarizada (TC) cardíaca en la que se observaron extensas calcificaciones pericárdicas que involucraban grasa epicárdica e infiltraban miocardio (Vídeo 2, Vídeo 3 y Figura 3). Se descartó etiología reumatológica, con marcadores (complemento, FR, ANA, AC doble cadena, AC anticardiolipina) negativos. Presentó aumento de los niveles de proteína $C$ reactiva ultrasensible, lo cual sugería la presencia de un proceso inflamatorio. Se descartó infección viral por virus de hepatitis $B$, virus de hepatitis $C$ y virus de la inmunodeficiencia humana. Se decidió manejo médico por su médico tratante, manteniéndose asintomático actualmente.

\section{Estudio por imagen}

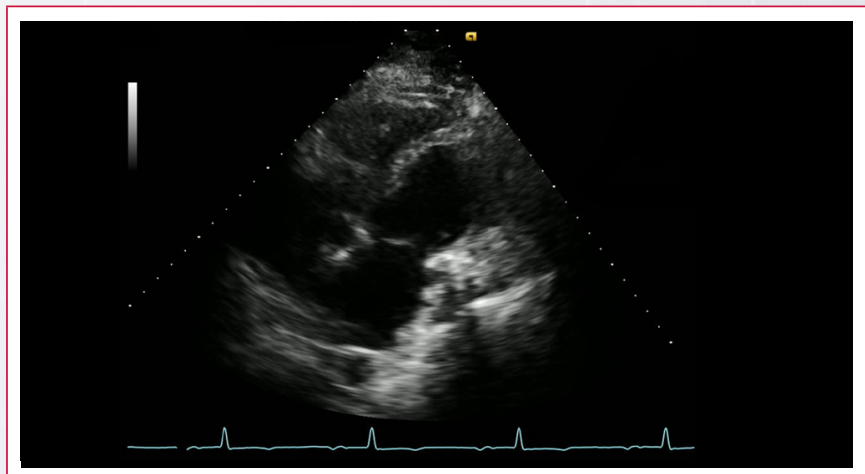

Vídeo 1. ETT bidimensional que muestra el movimiento de rebote septal característico en pericarditis constrictiva 


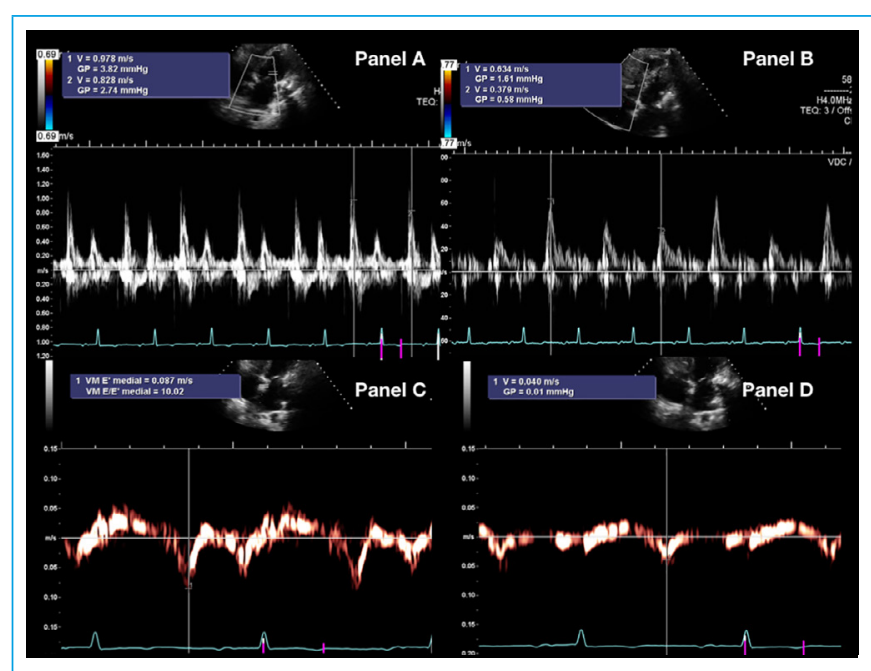

Figura 1. Panel A: patrón de llenado mitral en ETT que muestra variabilidad respiratoria exagerada. Panel B: patrón de llenado tricuspídeo en ETT que muestra variabilidad respiratoria exagerada. Panel C: Doppler tisular del anillo mitral medial que muestra velocidad de onda $E^{\prime}>8 \mathrm{~cm} / \mathrm{s}$. Panel $\mathbf{D}$ : Doppler tisular del anillo mitral lateral que muestra velocidad de onda $E^{\prime}$ menor que la medial (annulus reversus)

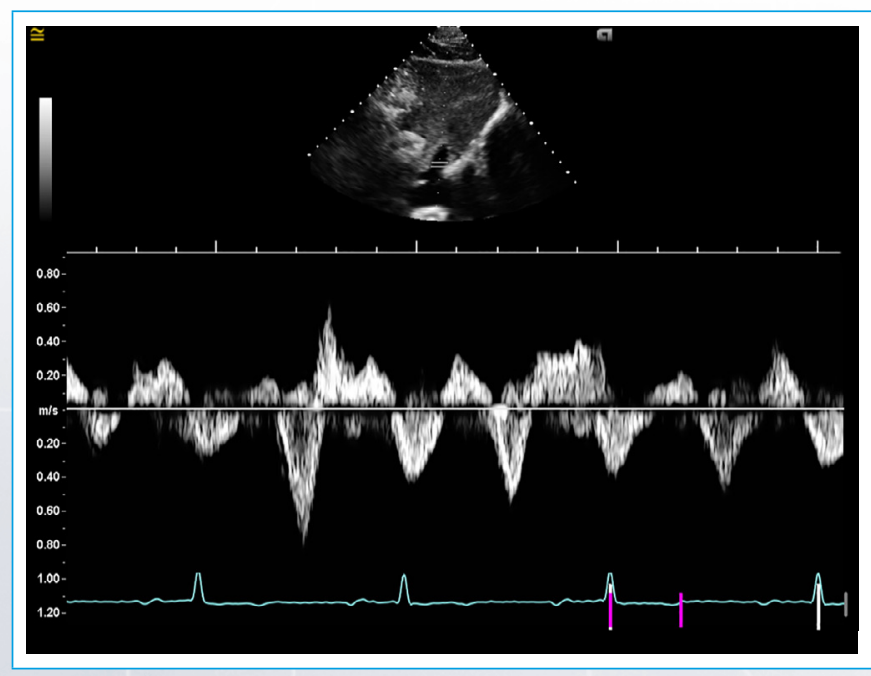

Figura 2. ETT, ventana subcostal, explorando venas suprahepáticas, donde se aprecia predominio del flujo reverso diastólico

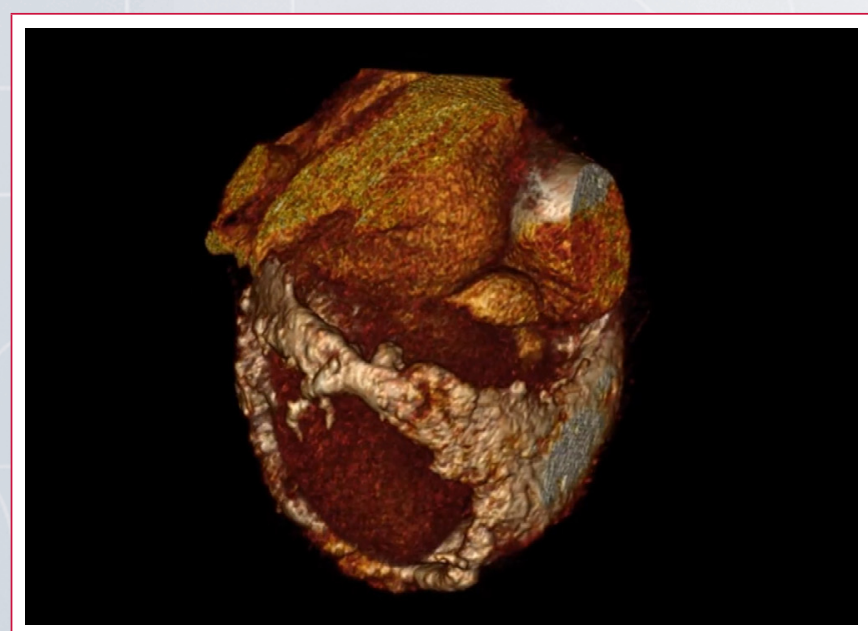

Vídeo 2. Angiotomografía cardíaca tridimensional, reconstrucción 3D, que muestra localización, disposición y extensión de la calcificación pericárdica

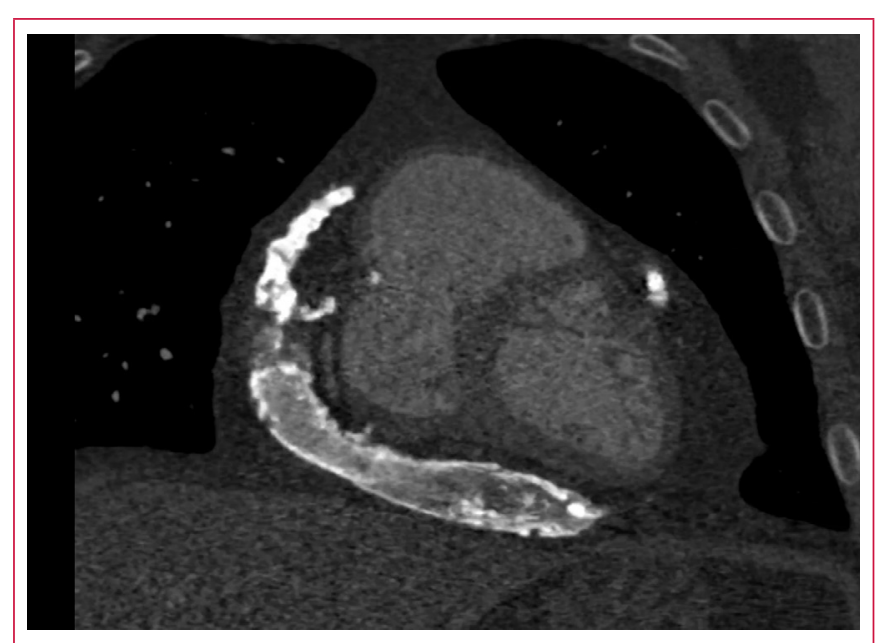

Vídeo 3. Angiotomografía cardíaca, cortes coronales, que muestran densidad y grosor de calcificaciones pericárdicas y su extensión hasta tejido miocárdico

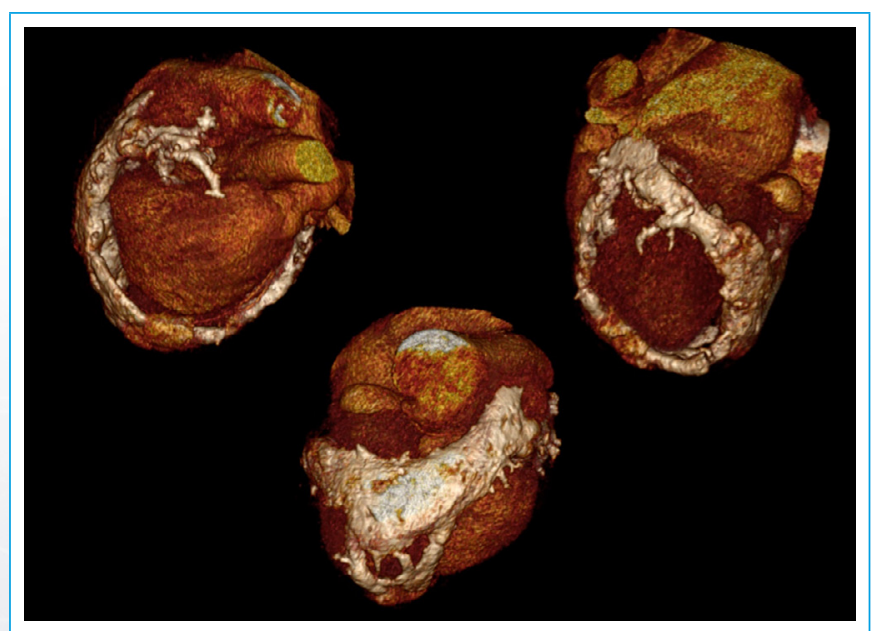

Figura 3. Angiotomografía cardíaca modo tridimensional, reconstrucción 3D. Detalle de la extensión de la calcificación pericárdica

\section{Caso clínico 2}

Varón de 48 años con palpitaciones, disnea y síndrome consuntivo. Clínicamente con insuficiencia cardíaca, fue referido a hospitalización. A su ingreso, se presentaba con hipotensión, taquicardia, ingurgitación yugular, signo de Kussmaul, edema de miembros inferiores y palpitaciones.

El electrocardiograma revelaba flutter auricular 2:1 con frecuencia cardíaca de $110 \mathrm{lpm}$. Se realizó angiotomografía pulmonar por sospecha de tromboembolismo pulmonar, encontrándose engrosamiento pericárdico con calcificaciones pericárdicas (Figura 4, Vídeo 4 y Vídeo 5).

En el ETT se apreció el pericardio engrosado, rebote septal, relación E/A mitral menor de 1.5, vena cava inferior de $28 \mathrm{~mm}$, onda e' medial anular mitral de 13 $\mathrm{cm} / \mathrm{s}$, annulus reversus, variación de flujo aórtico del $17 \%$ con la respiración, alteraciones regionales de la contractilidad ventricular izquierda de predominio lateral con fracción de eyección del $42 \%$, con lo que se hizo el diagnóstico de pericarditis constrictiva. Se realizó cateterismo cardíaco, observándose curva de presión auricular con onda "y" prominente, curva de presión en raíz cuadrada, con igualación de presiones diastólicas ventriculares. Se documentaron baciloscopias positivas para tuberculosis. 


\section{Caso clínico 3}

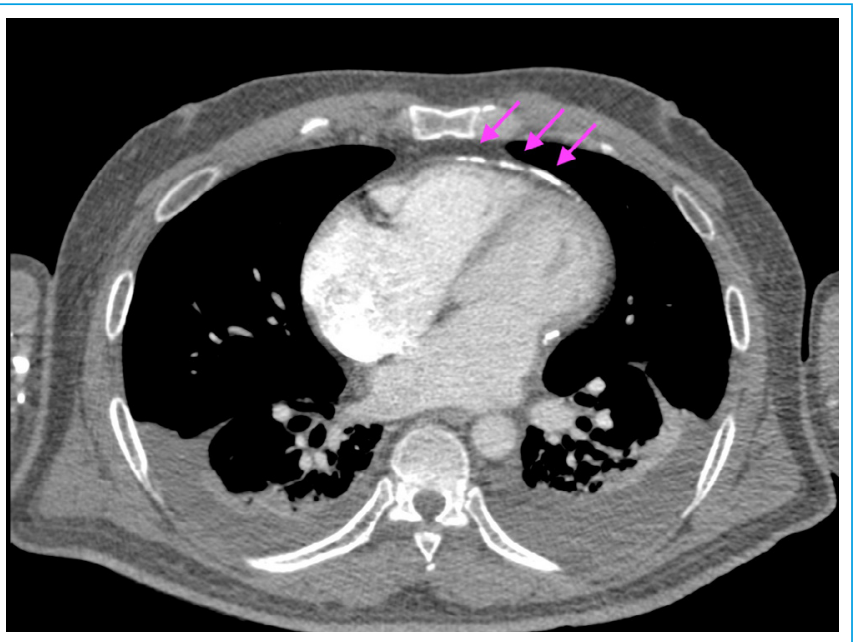

Figura 4. Angiotomografía cardíaca que muestra calcificaciones en pericardio anterior (flechas)

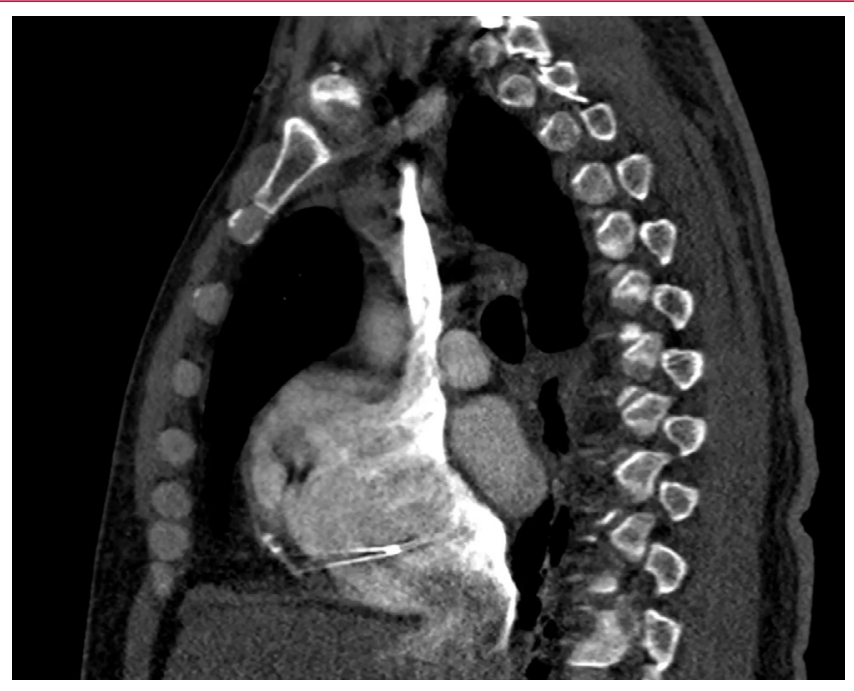

Vídeo 4. Angiotomografía cardíaca, cortes sagitales, que muestra calcificaciones pericárdicas de predominio anterior

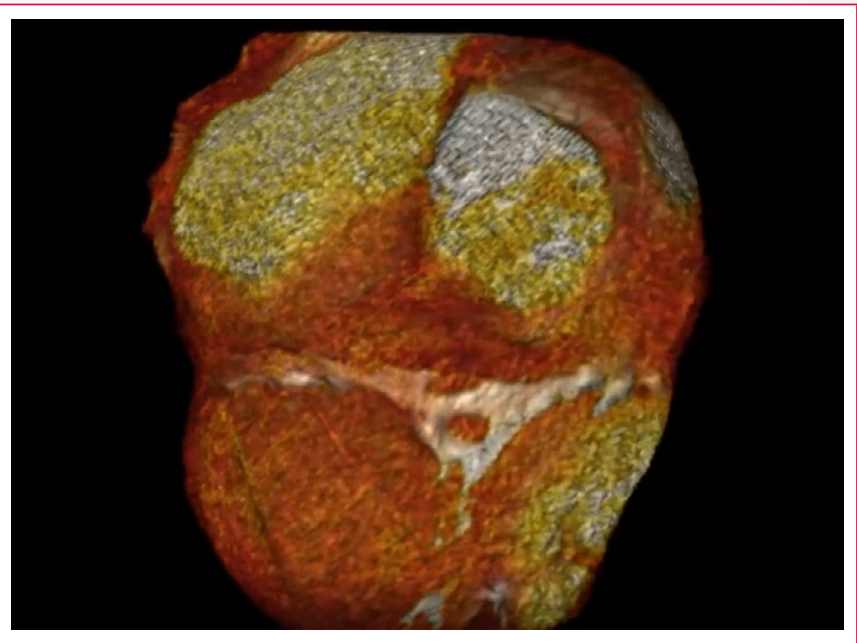

Vídeo 5. Angiotomografía cardíaca tridimensional, volume rendering, que muestra localización y disposición de las calcificaciones pericárdicas
Varón de 69 años con antecedente de tabaquismo. Inicia con disnea progresiva, edema de miembros inferiores y pérdida de peso (10 kg en 6 meses). Durante su estudio, se realizó tomografía de tórax que evidenció derrame pericárdico y engrosamiento pericárdico de $5 \mathrm{~mm}$, así como engrosamiento y derrame pleural derecho.

El ETT mostró un ventrículo izquierdo con fracción de eyección del 55\%, contractilidad regional en reposo normal y derrame pericárdico circunferencial severo con máxima separación de hojas pericárdicas de $35 \mathrm{~mm}$ anterior al ventrículo derecho, sin colapso de cavidades (Figura 5); patrón de llenado mitral restrictivo con variabilidad del 30\% (Figura 6); onda E' anular mitral medial de más de $8 \mathrm{~cm} / \mathrm{s}$ (Figura 7); y vena cava inferior de $28 \mathrm{~mm}$ con colapso inspiratorio insuficiente (Figura 8), por lo que se hizo el diagnóstico de pericarditis efusivo-constrictiva.

En la analítica de laboratorio, se evidenció BNP de $129 \mathrm{ng} / \mathrm{dl}$, troponina I 0.04 $\mathrm{ng} / \mathrm{dl}$, marcadores tumorales (ACE, APF, APE, Ca 125, Ca 19.9) negativos, marcadores inmunologicos (ANCA C, P, ANA, anti-DNA) negativos, perfil tiroideo normal y se descartó tuberculosis.

Se realizó pericardiectomía, liberando los grandes vasos del pericardio parietal con resección de pericardio de toda la superficie cardíaca hasta nivel de frénicos; se encontró pericardio parietal con engrosamiento de $5 \mathrm{~mm}$. El reporte de patología fue de fibrosis e inflamación crónica.

\section{Estudio por imagen}

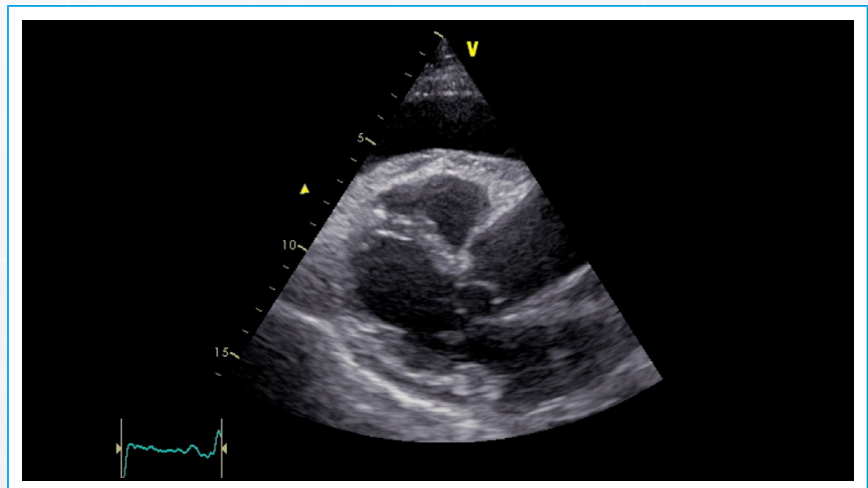

Figura 5. ETT, eje largo paraesternal, donde se observa derrame pericárdico severo

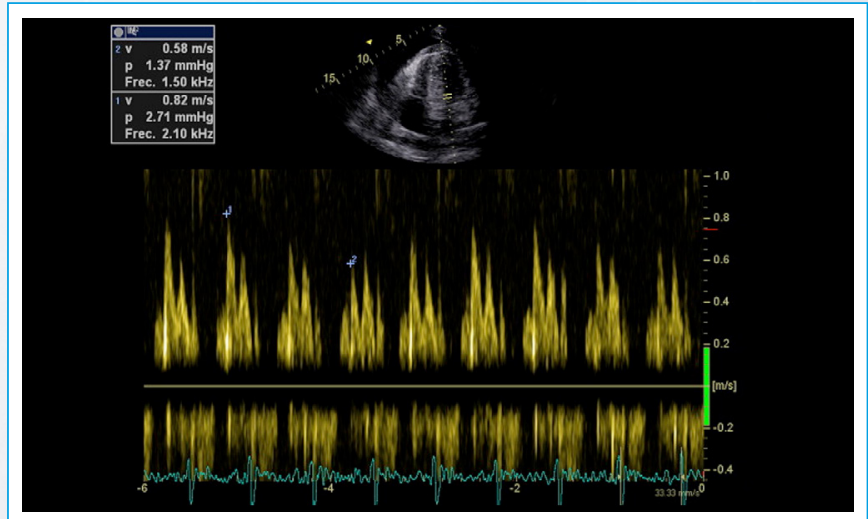

Figura 6. ETT, exploración con Doppler pulsado del flujo de llenado mitral, donde se observa variabilidad significativa de $30 \%$ 


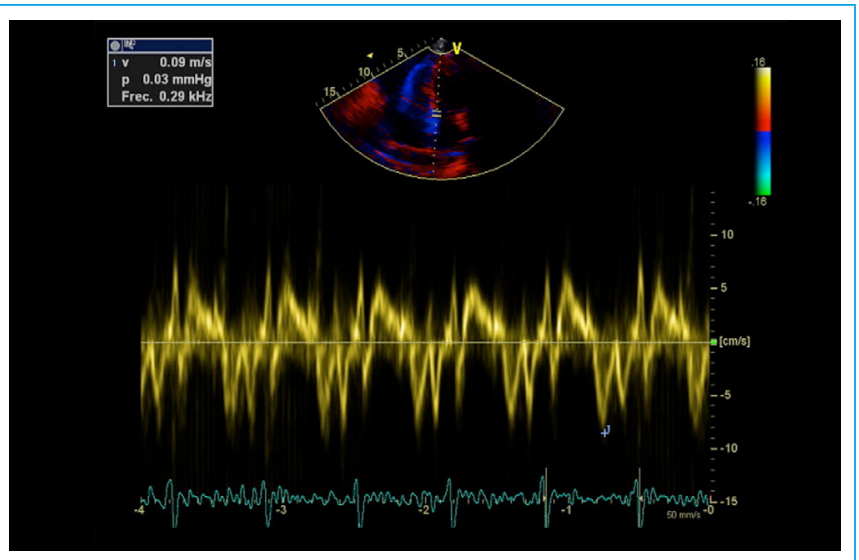

Figura 6. ETT, exploración con Doppler tisular anular medial, que muestra velocidad de onda $E^{\prime}$ de $9 \mathrm{~cm} / \mathrm{s}$

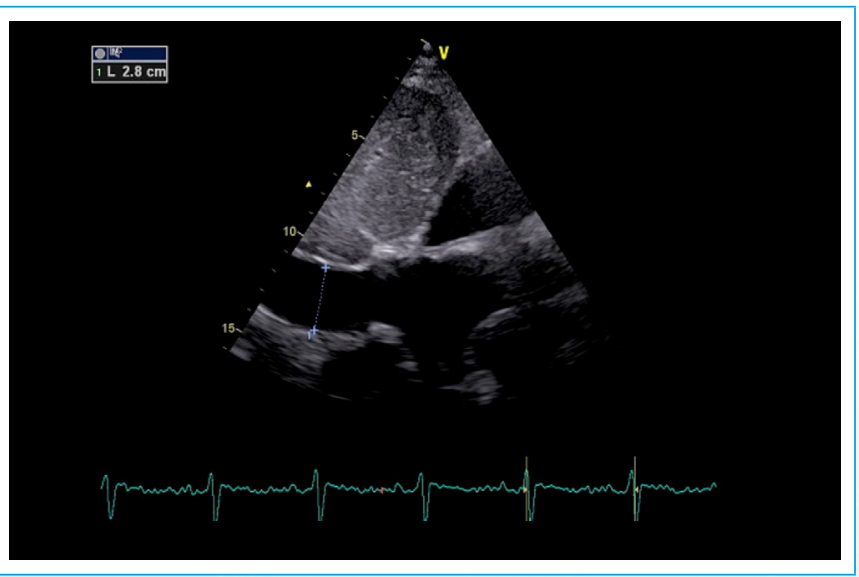

Figura 7. ETT, vista subcostal, donde se observa dilatación significativa de vena cava inferior

\section{Discusión}

El pericardio es un determinante del llenado ventricular. El aumento del líquido entre sus capas o su engrosamiento disminuyen la distensibilidad y causan cambios en la mecánica ventricular ${ }^{(1)}$. En la pericarditis constrictiva, el pericardio se encuentra engrosado (> $4 \mathrm{~mm}$ ) en un $80 \%$ de los casos. Los síntomas suelen ser de insuficiencia cardíaca derecha (disnea, distensión abdominal, ascitis, e ingurgitación yugular) $)^{(2)}$

El ecocardiograma confirma el diagnóstico en el 70\% de los casos. Las características ecocardiográficas son: aumento del grosor pericárdico, rebote septal, plétora de vena cava inferior, patrón restrictivo en llenado ventricular, velocidad de onda E transmitral con disminución superior al 25\% con la inspiración, velocidad de onda E transtricuspídea con disminución superior al $40 \%$ con la espiración, onda e' medial anular mitral $>8 \mathrm{~cm} / \mathrm{s}$ que condiciona el fenómeno de annulus reversus. La sensibilidad, especificidad y valor predictivo positivo del ecocardiograma para diferenciar una pericarditis constrictiva de una miocardiopatía restrictiva es del $81 \%, 100 \%$ y $100 \%$, respectivamente.

La TC cardíaca es de utilidad para evaluar el grosor del pericardio y detectar calcificaciones pericárdicas, así como para el abordaje quirúrgico y la evaluación de estructuras adyacentes ${ }^{(3-5)}$. La resonancia magnética (RM) cardíaca permite evaluar datos de constricción y restricción, siendo necesaria en casos en los que se observan patrones mixtos y cuando existe sospecha de infiltración miocárdica(6,7).
En el $25-50 \%$ de los casos de pericarditis constrictiva existen calcificaciones pericárdicas cuya etiología es generalmente idiopática, como en el primer caso que mostramos ${ }^{(8,9)}$. Además de las calcificaciones pericárdicas, se observan calcificaciones miocárdicas y, entre las causas de éstas, se incluyen isquemia, inflamación (enfermedades reumáticas, sarcoidosis, sepsis, etc.), infección (miocarditis, tuberculosis, equinococosis), trauma (cirugía, cardioversión, irradiación, hemorragia), neoplasias, enfermedad renal, hiperparatiroidismo, drogas, e idiopática. Los casos que muestran calcificación miocárdica abundante, que incluyen una o más cavidades cardíacas, e infiltran grandes vasos, generalmente son de origen idiopático, como lo fue en uno de nuestros $\operatorname{casos}^{(10)}$.

El tratamiento de elección es la pericardiectomía temprana, ya que tiene mejores desenlaces tempranos y tardíos, ya que la constricción prolongada causa atrofia miocárdica, constricción residual y falla cardíaca persistente, aun posterior a la pericardiectomía.

Está en discusión del beneficio de la pericardiectomía en pacientes con cambios crónicos, por el aumento leve o moderado en el volumen cardíaco. Este procedimiento puede ser perjudicial en miocardiopatía dilatada o disfunción severa del ventrículo derecho. El tratamiento no quirúrgico se basa en el uso de diuréticos, nitratos, inhibidores de la enzima convertidora de angiotensina, y terapia de resincronización ${ }^{(1,2,5,8-10)}$.

La Figura 9 muestra un algoritmo diagnóstico de imagen multimodal en pericarditis constrictiva.

\section{Conclusión}

Los casos presentados de pericarditis constrictiva muestran la dificultad diagnóstica de esta patología; los datos clínicos son inespecíficos, y en uno de ellos las alteraciones pericárdicas se encontraron como hallazgo en la TC. Además, en la pericarditis constrictiva, las etiologías son múltiples y no relacionadas. De aquí la importancia de aumentar la sospecha de esta enfermedad en pacientes con datos clínicos de insuficiencia cardíaca y síndrome consuntivo, especialmente en nuestro medio, así como solicitar la evaluación ecocardiográfica que permita orientar el diagnóstico para complementar posteriormente con otros estudios de imagen, como TC cardíaca, RM y/o cateterismo cardíaco.

La pericarditis constrictiva es una patología poco común, que puede ser tratada con éxito una vez diagnosticada, a diferencia de la miocardiopatía restrictiva. La ecocardiografía permite la caracterización fisiopatológica, mientras que la TC cardíaca ayuda a la caracterización anatómica del pericardio, a evaluar las estructuras adyacentes, y a definir el acceso a la resección quirúrgica del pericardio afectado. En casos no concluyentes o mixtos, la RM ayuda a diferenciar entre pericarditis constrictiva y miocardiopatía restrictiva.

\section{Ideas para recordar}

- La pericarditis constrictiva causa alteraciones en el pericardio que alteran la función cardíaca.

- Las principales causas en nuestro medio son tuberculosis, cirugía cardíaca y radiación; posteriormente, idiopática.

- El diagnóstico es fácil si existe alta sospecha clínica, considerando además los criterios ecocardiográficos que reportan alta sensibilidad y especificidad.

- La TC cardíaca permite observar las características del pericardio y definir su resección quirúrgica.

- La RM permite detectar casos mixtos entre constricción y restricción, así como definir infiltración miocárdica. 


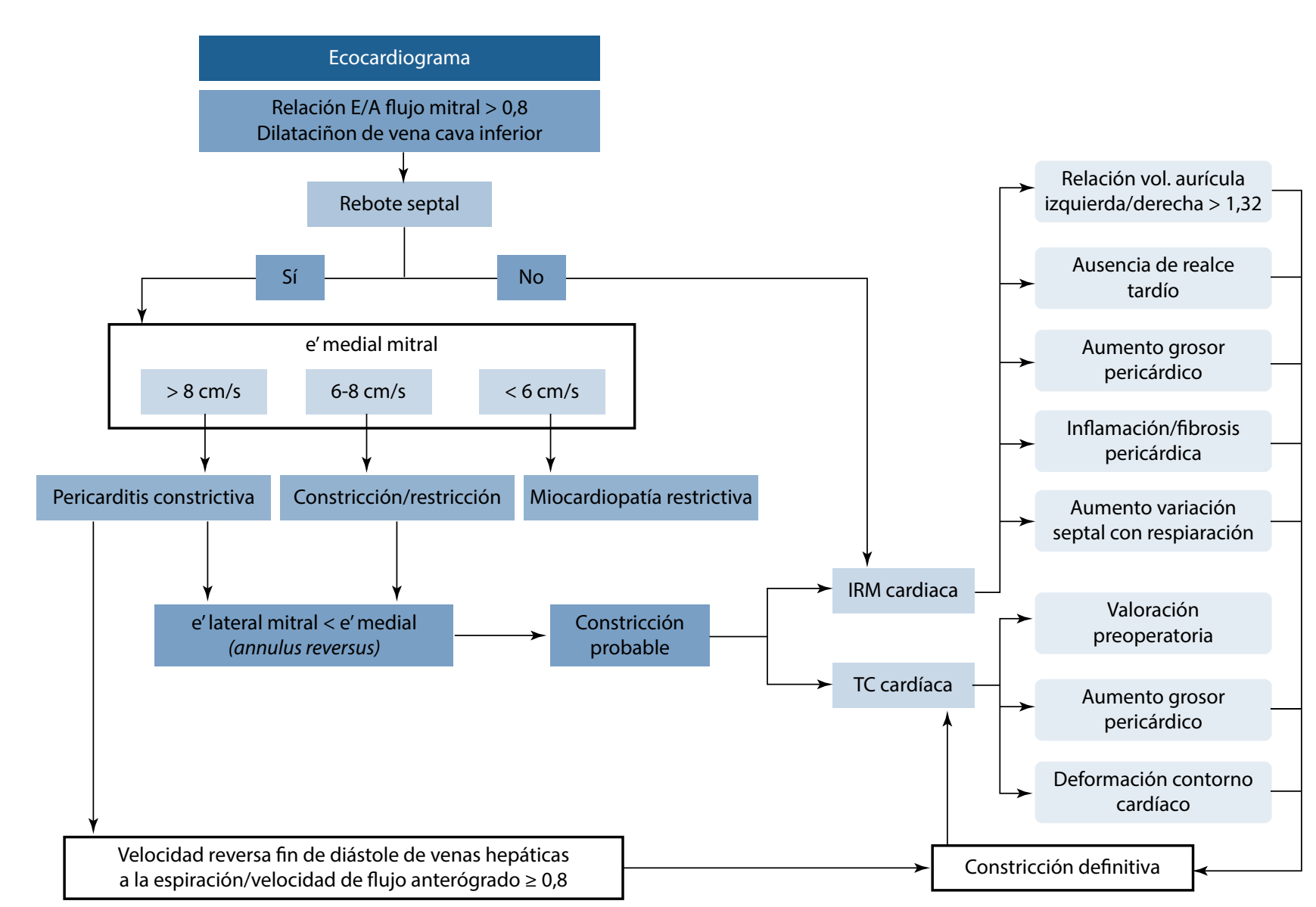

Figura 4. Algoritmo modificado de Welch ${ }^{(6)}$ y Geske et al. ${ }^{(7)}$ para el diagnóstico de pericarditis constrictiva

\section{Bibliografía}

1. Borlaug BA, Reddy YNV. The role of pericardium in heart failure. JACC Heart Fail 2019; 7: 574-585.

2. Tuck BC, Towsley MM. Clinical update in pericardial diseases. J Cardiothorac Vasc Anesth 2019; 33 (1): 184-199.

3. Kligerman S. Imaging of pericardial disease. Radiol Clin N Am 2019; 57: 179199.

4. Xu B, Harb SC, Klein AL. Utility of multimodality cardiac imaging in disorders of the pericardium. Echo Res Pract 2018; 5 (2): R37-R48.

5. Cosyns B, Plein S, Nihoyanopoulos $P$, Smiseth O, Achenbach S, Andrade MJ, et al. European Association of Cardiovascular Imaging (EACVI) position paper: multimodality imaging in pericardial disease. Eur Heart J 2015; 16 : $12-31$.
6. Welch TD. Constrictive pericarditis: diagnosis, management and clinical outcomes. Heart 2018; 104: 725-731.

7. Geske JB, Anavekar NS, Nishimura RA, Oh JK, Gersh BJ. Differentiation of constriction and restriction. J Am Coll Cardiol 2016; 68: 2329-2347.

8. Paniagua González M, Sánchez Alegre ML. Extensive pericardial calcification secondary to radiotherapy, causing mixed constrictive-restrictive pathology. BJR Case Rep 2017; 3: 00-36.

9. Ohri R, Salhiyyah K, Harding S, Ohri S. Pericardial calcification. A case report of a three-dimensional disease. Int J Surg Case Rep 2019; 57: 152-154.

10. Nance JW, Crane GM, Halushka MK, Fishman EK, Zimmerman SL. Myocardial calcifications; pathophysiology, etiologies, differential diagnoses, and imaging findings. J Cardiovasc Comp Tomogr 2015; 9: 58-67. 